\title{
Improving Quality of US Health Care Hinges on Improving Language Services
}

\author{
Risa Lavizzo-Mourey, MD, MBA \\ The Robert Wood Johnson Foundation, Princeton, NJ, USA.
}

J Gen Intern Med 22(Suppl 2):279-80

DOI: $10.1007 / \mathrm{s} 11606-007-0382-2$

(c) Society of General Internal Medicine 2007

$\mathrm{O}$ $\mathrm{n}$ behalf of the Robert Wood Johnson Foundation, I am delighted to support this supplement on language services. I believe that the quality of health care for all Americans will not be improved without a concentrated effort to ensure that people with limited English-speaking proficiency have access to language services and assistance in clinical encounters within America's hospitals and health systems.

In 1999, Congress asked the Institute of Medicine (IOM) to analyze disparities in our health care system, based on differences in patient race and ethnicity, which can sometimes be accompanied by language barriers. I was pleased to serve on the authors' panel. The resulting study, Unequal Treatment: Confronting Racial and Ethnic Disparities in Health Care, showed that patients of different ethnicities frequently received different levels of care, even when all other factors, such as income, health status, and insurance coverage were equal. The differences in care these patients received were attributed to many different factors, including whether or not patients and providers communicated effectively.

Eight years later, nearly one in five people in the USA now speak a language other than English at home, and while most can comfortably speak English when needed, experts estimate that as many as 20 million people in America-about one in every 15 people in the USA-speak and understand little, if any, English. ${ }^{1}$ As America becomes increasingly multilingual, physicians and other health care providers face a tremendous challenge-one that grows greater each year: How will we communicate efficiently and cost-effectively with patients who speak or understand limited English?

Because high-quality, patient-centered health care is contingent upon patients' understanding and following their doctors' advice-and upon health care providers listening to and understanding the needs of their patients-the stakes for meeting this challenge could not be higher. If we are unsuccessful, the result will be even bigger gaps in the quality of care that is provided to millions of non-English-speaking patients. We will have missed the challenge called forth in the IOM report and failed ourselves as health care leaders. That is why more and more providers are trying to improve the quality of language services that they provide-because they know it is directly linked to the quality of medical care their patients receive.

Language barriers impair discussions of symptoms and recommended therapies, resulting in misdiagnoses or poor treatment decisions. Communication barriers also impede adherence to treatment regimes. Studies have shown that patients who need an interpreter but do not receive one are less likely to understand instructions for taking medications, receive less information on medication side effects, and experience lower satisfaction with their care. ${ }^{2,3}$ One survey found that, in the absence of an interpreter, Spanish-speaking patients with limited English proficiency reported significant difficulty in being able to fully explain their symptoms or trust the medical recommendations of a provider who only speaks English. ${ }^{4}$

I guess this should not come as a surprise. Even for the most fluent English speakers among us, navigating a health care environment can be an overwhelming experience. Physicians, nurses, technicians, and other health care professionals typically use unfamiliar medical terminology that may sound foreign regardless of primary language. For patients who speak and understand little or no English, these challenges increase exponentially. When patients and doctors cannot talk with each other about symptoms, diagnoses, test results, medications, treatment plans, and follow-up care, the result is bad medical care.

Far too often, health care providers rely on a patient's family member, friend, or even a member of the hospital's non-medical staff to communicate about clinical care-yet these individuals seldom have training in translating or interpreting medical terminology. Additionally, family members or friends may be reluctant to reveal some personal information about symptoms that could be embarrassing to the patient-information that may be critical to successful diagnosis and treatment. Even seemingly simple things, like requesting an additional blanket, relaying information about a food allergy, or asking about visiting hours can seem next to impossible.

For many years, the Robert Wood Johnson Foundation has worked to improve the quality of health care for all Americans. We recognize that achieving real quality for all requires special attention to the care delivered to racially and ethnically diverse patients in hospitals and other medical settings. Among many other efforts, the Foundation's commitment to both improving health care quality and reducing disparities in care has included:

- Helping hospitals identify and reduce disparities among patients with cardiovascular disease;

- Working with hospitals to develop language service performance measures that can test how well they meet the needs of patients with limited English proficiency;

- Advancing the work of local communities-many of which serve patients that are predominantly limited in English proficiency-to align providers, purchasers, and patients around common quality goals to raise the standard of care; 
- Working to build consensus in the medical community about reliable measures of quality for all patients-with special consideration for specific interventions needed for patients of color;

- Supporting work that explores how to structure and provide incentives that reward high-quality providers, and more.

Two of the Foundation's current programs focus specifically on the inherent link between providing quality care and providing quality language services for non-English-speaking patients. The Foundation's Hablamos Juntos program, which translates from Spanish to English as "We speak together," was first introduced in the fall of 2002 with the goal of developing affordable models to help English-speaking providers communicate more effectively with their Spanish-speaking patients. Introduced in November 2006, Speaking Together is a learning network of ten hospitals working collaboratively to develop and test the most effective and efficient ways to provide language services in clinical settings to patients with limited English proficiency.

The articles featured in this supplement give me hope. They address the critical issue of language barriers in health care in-depth and at a breadth that truly breaks new ground. I am proud that, perhaps more than ever before, leading researchers across the country are applying their skills to identifying solutions to language barriers that affect the quality of patient care.

In the coming months, the Foundation will unveil a new effort that will focus on regional strategies to improve health care quality for all. Its aim will be to help communities set and achieve ambitious goals to improve care in ways that matter to all patients and their families and, in particular, to patients from specific racial and ethnic backgrounds who often experience lower-quality care.

Eight years after the landmark IOM report on disparities in health care, I continue to believe that it will take a network of coordinated, community-based strategies to ensure that
English-speaking and non-English-speaking patients alike receive the highest quality of health care available. That is something that should be automatically assumed in a country that spends more on health care than any other nation in the world. We have a long way to go, but, as these articles highlight, progress is indeed being made.

The Robert Wood Johnson Foundation focuses on the pressing health and health care issues facing our country. As the nation's largest philanthropy devoted exclusively to improving the health and health care of all Americans, the Foundation works with a diverse group of organizations and individuals to identify solutions and achieve comprehensive, meaningful, and timely change. For more than 35 years, the Foundation has brought experience, commitment, and a rigorous, balanced approach to the problems that affect the health and health care of those it serves. When it comes to helping Americans lead healthier lives and get the care they need, the Foundation expects to make a difference in your lifetime. For more information, visit http://www.rwif.org.

Corresponding Author: Risa Lavizzo-Mourey, MD, MBA; The Robert Wood Johnson Foundation, P.O. Box 2316, College Road East and Route 1, Princeton, NJ 08543, USA (e-mail: oped@rwif.org).

\section{REFERENCES}

1. US Bureau of the Census. Profile of Selected Social Characteristics: 2000 (Table DP-2, Matrices P19, P20, PCT13, and PCT14). Retrieved August 28, 2007 from http://www.factfinder.census.gov/servlet/MetadataBrowserServlet? type=dataset\&id=DEC_2000_SF3_U\&_lang=en.

2. Andrulis D, Goodman N, Pryor N. What a difference an interpreter can make: health care experiences of uninsured with limited English proficiency. Boston, MA: The Access Project; April 2003.

3. David RA, Rhee B. The impact of language as a barrier to effective health care in an underserved urban Hispanic community. Mt Sinai J Med. 1998;65(5,6):393-397.

4. Wirthlin Worldwide. Hablamos Juntos: We Speak Together, Final Report (2001). Retrieved August 22, 2007 from http://www.hablamosjuntos.org/ pdf_files/wirthlin_report.pdf. 